\title{
Pulmonary metastases of adult Wilms' tumor 17 years after the primary site
}

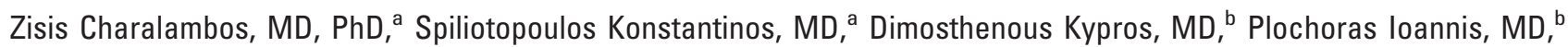 \\ Pantelidaki Aikaterini, MD, ${ }^{\mathrm{b}}$ and Bellenis Ion, MD, PhD, FETCS, ${ }^{a}$ Athens, Greece
}

Supplemental material is available online.

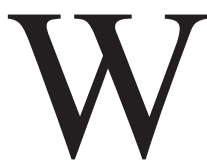

ilms' tumor accounts for most childhood renal neoplasms, and it is the third most common solid cancer in children under the age of 10 years. However, it infrequently occurs in adults, and only sporadic reports of isolated adult Wilms' tumor appear in the literature. ${ }^{1}$

\section{Clinical Summary}

A 47-year-old woman was admitted to our hospital after a chest radiograph showed a big shadow in the upper left lung (Figure E1). Chest computed tomography (CT) confirmed a mass sized $7 \times 10 \times$ $8 \mathrm{~cm}$ that was located in the left upper lobe (LUL) with atelectasis of the LUL (Figure 1) and revealed enlargement of the ipsilateral lymph nodes and secondary nodules in the left lower lobe and the right lower lobe at a maximum diameter of $3 \mathrm{~cm}$. Thrombocytopenia that had started 2 months previously initiated diagnostic investigation. The patient was an ex-smoker (20 packets-years), with a history of left nephrectomy 17 years previously because of a stage II Wilms' tumor. Transbronchial needle aspiration through the left upper lobar bronchus set the diagnosis of metastatic Wilms' tumor with relatively good differentiation. The complementary diagnostic work-up (abdominal and brain CT scan and skeletal bone scanning) did not detect any further lesions, and the patient underwent 2 cycles of chemotherapy. A new chest CT scan found a decrease in the size of the findings in both lungs, which was indicative of the response of the mass to the chemotherapy. Surgical excision of the tumor was decided, and a 2-stage procedure was chosen as appropriate. A left thoracotomy was performed first because of the size of the tumor in the LUL and the atelectasis. Left upper lobectomy and wedge resection of 2 lesions of the left lower lobe was required to remove all metastases from the left lung. Histologic examination revealed metastatic Wilms' tumor to the LUL and left lower lobe, infiltrating the lung parenchyma, the lobar bronchus, the pleura, and 3 peribronchial lymph nodes. The mediastinal lymph nodes were without invasion. Immunohistochemical

From the Departments of Thoracic and Vascular Surgery ${ }^{\mathrm{a}}$ and Pathology, ${ }^{\mathrm{b}}$ Evangelismos General Hospital, Athens, Greece.

Received for publication Jan 26, 2006; accepted for publication March 15, 2006.

Address for reprints: Charalambos Zisis, MD, 17A, Patriarchou Grigoriou str, 166 74-Glyfada, Greece (E-mail: chzisis@otenet.gr).

J Thorac Cardiovasc Surg 2006;132:199-200

$0022-5223 / \$ 32.00$

Copyright $\odot 2006$ by The American Association for Thoracic Surgery

doi:10.1016/j.jtcvs.2006.03.034 staining with neuron specific enolase (NSE) and vimentin was positive, whereas cytokeratin 7 (CK7), cytokeratin 20 (CK20), thyroid transcription factor-1 (TTF-1), and renal cell carcinoma (RCC) were negative (Figures 2, E2).

Fifteen days after the left thoracotomy, the patient underwent a right thoracotomy and wedge resections of 2 lesions, one from the upper and the second from the lower lobe, whereas the pathologic examination confirmed the diagnosis of the metastatic Wilms' tumor. The postoperative course was uneventful after both thoracotomies, and the patient was discharged on the fourth and third postoperative days, respectively.

The patient is currently doing well 1 month after the right thoracotomy, and close follow-up has been recommended.

\section{Discussion}

Classically, the prognosis of adult cases of Wilms' tumor was considered poorer than that in children, among whom $90 \%$ can be expected to become long-term survivors. ${ }^{2}$ However, there is no adequate experience in the ideal management of such patients because of the small number of adult cases. The treatment proposed includes a combination of all available modalities (surgical intervention, radiotherapy, and chemotherapy). ${ }^{1}$

The special interest of this particular case is that the patient presented with a Wilms' tumor in her adult life at the age of 30 years and that this tumor recurred distally after 17 years. Relapse most often occurs within 4 years of initial diagnosis, and the most common metastatic location is the lung. A special case recently reported concerns a solitary pulmonary lesion 20 years after Wilms' tumor primary resection at the age of 2 years. ${ }^{3}$ To our knowledge, such a late relapse case of an adult Wilms' tumor, especially in both lungs with more than one metastatic site and with invasion of the locoregional intrathoracic lymph nodes, has never been reported previously.

The general prognostic principle in pulmonary metastasectomies is that better results are obtained if the disease-free interval is long enough to justify a more aggressive approach in these specific cases. $^{4}$ This principle has been applied in our case to secure a potentially favorable prognosis to the patient.

\section{References}

1. Orditura M, De Vita F, Catalano G. Adult Wilms' tumor: a case report. Cancer. 1997;80:1961-5.

2. Thomas PR. Wilms' tumor: changing role of radiation therapy. Semin Radiat Oncol. 1997;7:204-11.

3. Parambil JG, Aughenbaugh GL, Pereira TC, Gillespie DJ, Ryu JH. Solitary pulmonary metastasis presenting 20 years after primary resection of Wilms tumor. Mayo Clin Proc. 2005;80:1514-6.

4. Torre W, Rodriguez-Spiteri N, Sierrasesumaga L. Current role for resection of thoracic metastases in children and young adults-do we need different strategies for this population? Thorac Cardiovasc Surg. 2004;52:90-5. 

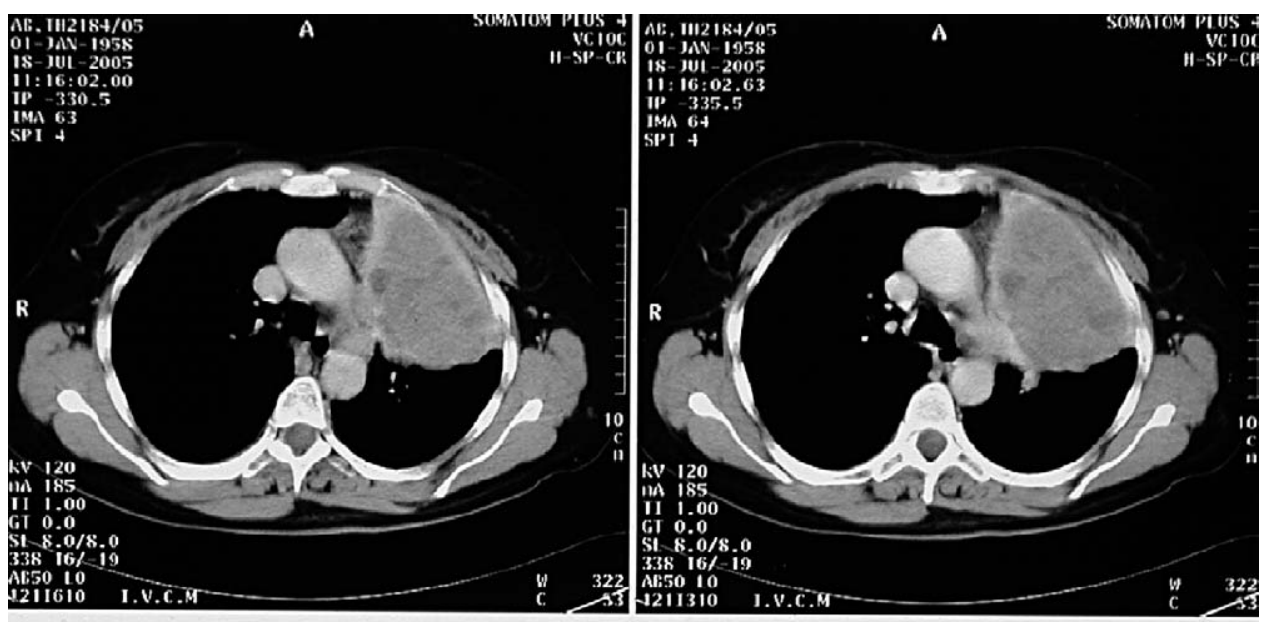

Figure 1. Preoperative chest computed tomographic scan demonstrating the metastasis of the left upper lobe, which is atelectatic.
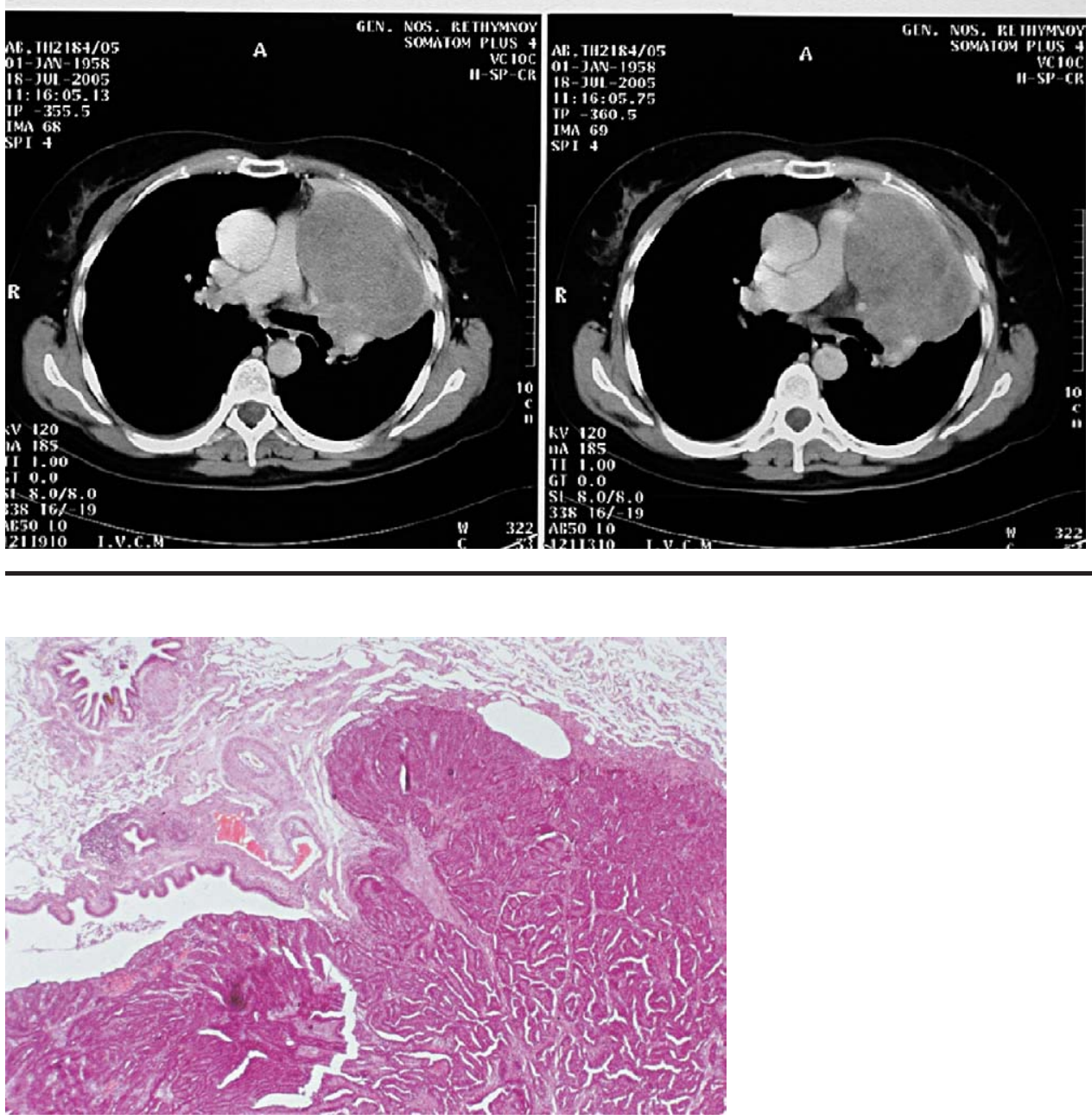

Figure 2. Characteristic morphologic features of the tumor. (Hematoxylin and eosin, original magnification $40 \times$.) 

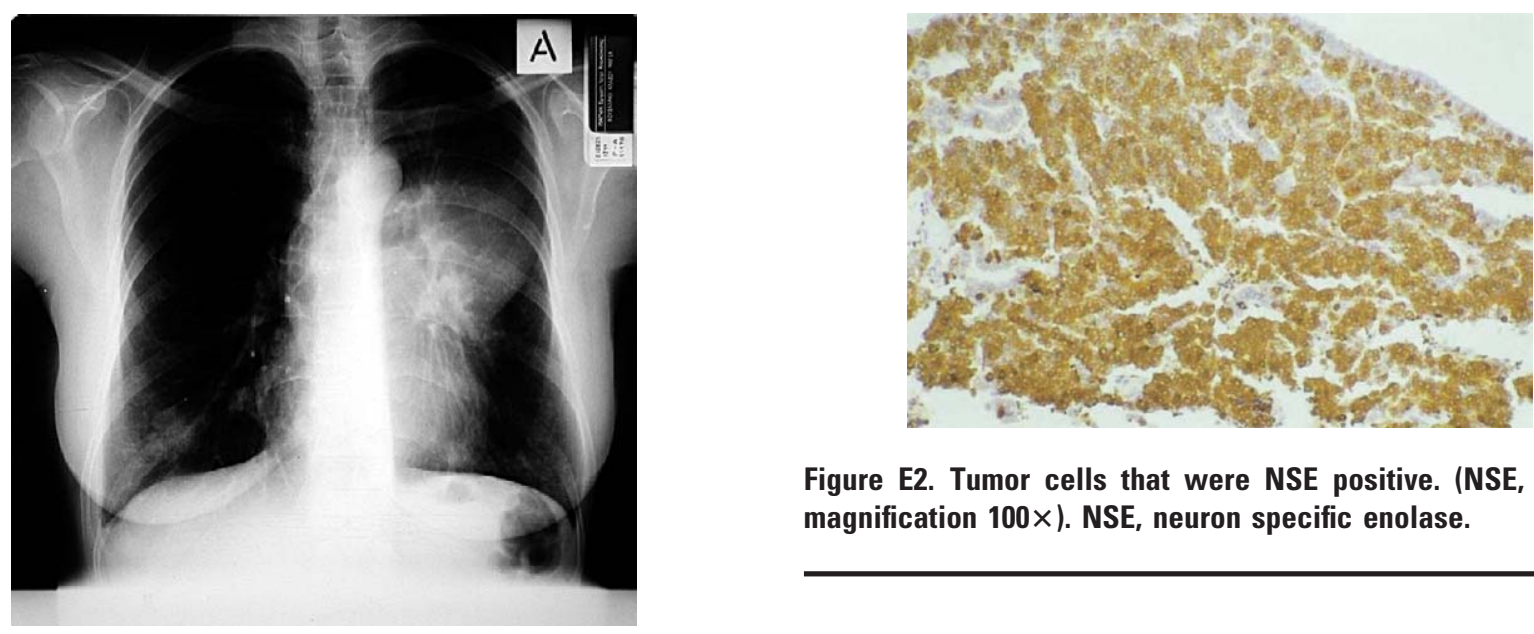

Figure E2. Tumor cells that were NSE positive. (NSE, original magnification $100 \times$ ). NSE, neuron specific enolase.

Figure E1. Preoperative chest radiograph showing the mass of the left upper lobe and the nodules of the lungs bilaterally representing secondary lesions. 